\title{
ITERATED FUNCTION SYSTEM QUASIARCS
}

\author{
ANNINA ISELI AND KEVIN WILDRICK
}

\begin{abstract}
We consider a class of iterated function systems (IFSs) of contracting similarities of $\mathbb{R}^{n}$, introduced by Hutchinson, for which the invariant set possesses a natural Hölder continuous parameterization by the unit interval. When such an invariant set is homeomorphic to an interval, we give necessary conditions in terms of the similarities alone for it to possess a quasisymmetric (and as a corollary, bi-Hölder) parameterization. We also give a related necessary condition for the invariant set of such an IFS to be homeomorphic to an interval.
\end{abstract}

\section{INTRODUCTION}

Consider an iterated function system (IFS) of contracting similarities $\mathcal{S}=$ $\left\{S_{1}, \ldots, S_{N}\right\}$ of $\mathbb{R}^{n}, N \geq 2, n \geq 1$. For $i=1, \ldots, N$, we will denote the scaling ratio of $S_{i}: \mathbb{R}^{n} \rightarrow \mathbb{R}^{n}$ by $0<r_{i}<1$. In a brief remark in his influential work [18, Hutchinson introduced a class of such IFSs for which the invariant set is a Peano continuum, i.e., it possesses a continuous parameterization by the unit interval. There is a natural choice for this parameterization, which we call the Hutchinson parameterization.

Definition 1.1 (Hutchinson, 1981). The pair $(\mathcal{S}, \gamma)$, where $\gamma$ is the invariant set of an IFS $\mathcal{S}=\left\{S_{1}, \ldots, S_{N}\right\}$ with scaling ratio list $\left\{r_{1}, \ldots, r_{N}\right\}$ is said to be an IFS path, if there exist points $a, b \in \mathbb{R}^{n}$ such that

(i) $S_{1}(a)=a$ and $S_{N}(b)=b$,

(ii) $S_{i}(b)=S_{i+1}(a)$, for any $i \in\{1, \ldots, N-1\}$.

Recall that the Hutchinson operator $T$ associated to an IFS is defined by $T(A)=$ $\bigcup_{i=1}^{N} S_{i}(A)$ for sets $A \subseteq \mathbb{R}^{n}$. For an IFS path, the image of the line segment connecting $a$ and $b$ under any iterate $T^{k}, k \in \mathbb{N}$, is a connected, piecewise linear set with a natural parameterization arising from the IFS $\mathcal{S}$. As $k$ tends to infinity, these parameterizations converge to the Hutchinson parameterization of the invariant set (see Section [5).

The canonical example of an IFS path is the Koch snowflake arc; however, in general, the invariant set of an IFS path need not be homeomorphic to the unit interval (e.g., the Peano space-filling arc). Characterizing the IFS paths for which this is the case seems to be a very difficult problem that displays chaotic behavior. Since the invariant set of an IFS does not determine the IFS, it seems that the most reasonable version of this task is to characterize the IFS paths for which the Hutchinson parameterization is injective (and hence a homeomorphism). As will be shown in Section 5 ( see in particular Proposition 5.2), the injectivity of the

Received by the editors November 20, 2015 and, in revised form, November 25, 2016.

2010 Mathematics Subject Classification. Primary 28A80, 30C65. 
Hutchinson parameterization can be easily reformulated in terms of the pair $(\mathcal{S}, \gamma)$ as follows:

Definition 1.2. We say that an IFS path $(\mathcal{S}, \gamma)$ is an IFS arc if

(i) $S_{i}(\gamma) \cap S_{i+1}(\gamma)=\left\{S_{i+1}(a)\right\}$ for $i \in\{1, \ldots, N-1\}$,

(ii) $S_{i}(\gamma) \cap S_{j}(\gamma)=\varnothing$ for $i, j \in\{1, \ldots, N-1\}$ with $|i-j|>1$,

where $a \in \mathbb{R}^{n}$ is as in Definition 1.1.

In many concrete examples, the invariant set $\gamma$ of an IFS arc is known to be a quasiarc, i.e., a quasisymmetric image of $[0,1]$. For an excellent introduction to quasisymmetric mappings; see the foundational article of Tukia and Väisälä [25] and the book [16]. An arc in $\mathbb{R}^{2}$ is a quasiarc if and only if the arc is the image of a compact interval under a quasiconformal self-mapping of $\mathbb{R}^{2}$. In fact, quasiarcs admit many characterizations [13, including by a simple geometric condition called bounded turning; see Section 2.2 for details. Let us say that an IFS arc is an IFS quasiarc if its invariant set is a quasiarc.

IFS quasiarcs play an important role in the theory of quasiconformal mappings, particularly with respect to questions about dimension distortion, and also appear in connection with Schwarzian rigid domains [3. For this reason, it is desirable to have a large, concrete family of IFS quasiarcs available for study and use. To those familiar with the theory of quasiconformal mappings, it may seem likely that every IFS arc is an IFS quasiarc due to the apparent self-similarity of the construction. However, this is not the case [26]:

Theorem 1.3 (Wen and $\mathrm{Xi}, 2003$ ). There is an IFS arc that is not a quasiarc.

Roughly speaking, the fact that the invariant set of an IFS arc is indeed an arc indicates that any obstruction preventing it from being a quasiarc can only occur infinitesimally and not globally (see Section 4); this seems impossible for a selfsimilar object. However, when the ratios $r_{1}$ and $r_{N}$ are not equal, one is not $a$ priori guaranteed that scaled copies of small pieces of the IFS arc also appear at large scale. In fact, this is the only obstacle, as we indicate in Section 5.5. Our main result gives a fairly large class of IFS arcs in $\mathbb{R}^{n}$ for which this obstacle does not occur. Aside from the assumption that the IFS path is an arc, the class is defined in terms of the similarities alone and the condition defining the class is simple to check.

Since the property of being a quasiarc in $\mathbb{R}^{n}$ is invariant under similarities, it is natural to assume that an IFS path $(\mathcal{S}, \gamma)$ in $\mathbb{R}^{n}$ is normalized so that the point $a$ fixed by $S_{1}$ is the origin and the point $b$ fixed by $S_{N}$ is $\mathbf{e}_{1}=(1,0, \ldots, 0) \in \mathbb{R}^{n}$. We recall that for each (contracting) similarity $S_{i}: \mathbb{R}^{n} \rightarrow \mathbb{R}^{n}$ of an IFS path $(\mathcal{S}, \gamma)$ there exists a ratio $r_{i} \in(0,1)$, an orthogonal transformation $A_{i}$, and a translation vector $b_{i} \in \mathbb{R}^{n}$ such that $S_{i}(x)=r_{i} A_{i}(x)+b_{i}$ for all $x \in \mathbb{R}^{n}$. Note that if $(\mathcal{S}, \gamma)$ is a normalized IFS path, then $b_{1}=0$.

Theorem 1.4. Let $(\mathcal{S}, \gamma)$ be a normalized IFS arc in $\mathbb{R}^{n}$ such that:

(A) There exist numbers $t, s \in \mathbb{N}$ such that $r_{1}^{t}=r_{N}^{s}$.

(B) Either $A_{1}^{t}=A_{N}^{s}$ or there exist numbers $p, q \in \mathbb{N}$ such that $A_{1}^{q}=A_{N}^{p}=\mathrm{Id}$, where Id denotes the identity matrix.

Then $(\mathcal{S}, \gamma)$ is an IFS quasiarc.

In Corollary 4.3, we will see that if $n=2$, then one can find plenty of easy-tocheck conditions that are sufficient for Theorem 1.4 to hold. 
Condition (A) is violated by the example of Wen and $\mathrm{Xi}$, and hence Theorem 1.4 fails without it. We do not know if Theorem 1.4 fails without condition $(B)$. However, we suspect that if condition (B) fails badly (see e.g. condition (2) in Theorem 1.7), then the path $\gamma$ has self-intersections and hence $(\mathcal{S}, \gamma)$ is not an IFS arc. Theorem 1.7 proves this conjecture for some cases of IFS paths in $\mathbb{R}^{2}$.

Besides the algebraic conditions of Theorem 1.4 we also briefly discuss a simpler and well-known condition for an IFS arc to be an IFS quasiarc, which we call the cone containment condition (see also 26). The cone containment condition is harder to check but covers many cases not covered by Theorem 1.4. However, not all IFS arcs satisfying the hypotheses of Theorem 1.4 satisfy the cone containment condition. Also note that neither the conditions in Theorem 1.4 nor the cone containment condition guarantee that an IFS path $(\mathcal{S}, \gamma)$ is actually a topological interval (see Remark 4.7).

A similar but slightly larger class of iterated function systems, called zippers, has been examined by Aseev, Tetenov, and Kravchenko in 11. There, the authors give a different collection of conditions on the IFS that guarantee that the invariant set is a quasiarc; there seems to be no overlap between those results and Theorem 1.4 . Other subclasses of zippers have been considered in connection with quantitative dimension distortion of planar quasiconformal mappings; in this case holomorphic motions can be used to show the quasiarc property 2. A wonderful visualization for such function systems can be found at 22 . In neither of these works are the zippers considered actually IFS paths, although in some cases the invariant set of the zipper can also be realized as the invariant set of an IFS path.

Theorem 1.4 provides a large, concrete class of IFS quasiarcs. We also show that IFS quasiarcs have special properties not shared by all quasiarcs nor by all IFS arcs. For example, IFS quasiarcs have particularly nice parameterizations:

Theorem 1.5. Let $(\mathcal{S}, \gamma)$ be an IFS arc with similarity dimension s. Then $(\mathcal{S}, \gamma)$ is an IFS quasiarc if and only if the Hutchinson parameterization of $\gamma$ by the interval $[0,1]$ is $\frac{1}{s}$-bi-Hölder continuous.

We recall that a metric space $(X, d)$ is Ahlfors $s$-regular, $s \geq 0$, if there is a constant $K \geq 1$ such that for each $x \in X$ and $0<r<2 \operatorname{diam} X$,

$$
\frac{r^{s}}{K} \leq \mathscr{H}^{s}(B(x, r)) \leq K r^{s}
$$

where $\mathscr{H}^{s}$ denotes $s$-dimensional Hausdorff measure. A $\frac{1}{s}$-bi-Hölder continuous image of $[0,1]$ is Ahlfors $s$-regular, and so Theorem 1.5 implies that if $(\mathcal{S}, \gamma)$ is an IFS quasiarc with similarity dimension $s$, then $\gamma$ is Ahlfors $s$-regular. In particular, the similarity dimension $s$ of $(\mathcal{S}, \gamma)$ is equal to the Hausdorff dimension of $\gamma$.

An immediate consequence of Theorem 1.5 is the following somewhat surprising statement:

Corollary 1.6. Let $\alpha$ and $\beta$ be IFS quasiarcs with equal similarity dimension. Then $\alpha$ and $\beta$ are bi-Lipschitz equivalent.

Statements such as Corollary [1.6 are referred to in the literature as Lipschitz equivalence results and are ubiquitous; see, for example, [11], [12, [27, [19, as well as [9] and 23] and the references therein. 
The surprising nature of Corollary 1.6 is illustrated by the fact that the four arcs depicted in Figure 1 represent bi-Lipschitz equivalent quasiarcs (metrized as subsets of $\mathbb{R}^{2}$ ) of similarity and Hausdorff dimension $s=\frac{\log 4}{\log 3}$; see Sections 4.2 and 5.4 for the precise definitions of these arcs.

As pointed out by Wen 26, Theorem 1.3 implies that Corollary 1.6 fails for the class of IFS arcs. Moreover, it is not difficult to find (non-IFS) quasiarcs for which the statement fails for Hausdorff dimension.

While Theorem 1.5 indicates that the class of IFS quasiarcs is much smaller than the class of all quasiarcs, an important result of Rohde [24] and its generalization by Herron and Meyer [17] indicates that every quasiarc can be obtained, up to a bi-Lipschitz mapping, by a snowflake-type construction.

One may (reasonably) complain that it is difficult to know if the invariant set of an IFS path is an arc. However, there are criteria, similar in spirit to those of Theorem 1.4, that guarantee an IFS path in $\mathbb{R}^{2}$ is not an arc. The angles $\alpha_{1}$ and $\alpha_{N}$ appearing in the statement below are the angles of counterclockwise rotation provided by the orthogonal transformations $A_{1}$ and $A_{N}$ of $\mathbb{R}^{2}$ associated to $S_{1}$ and $S_{N}$.

Theorem 1.7. Let $(\mathcal{S}, \gamma)$ be a normalized IFS path in $\mathbb{R}^{2}$ such that

(1) $S_{1}$ and $S_{N}$ are orientation preserving,

(2) there exist numbers $t, s \in \mathbb{N}$ such that $r_{1}^{t}=r_{N}^{s}$, and

$$
\left(t \alpha_{1}-s \alpha_{N}\right) \in 2 \pi(\mathbb{R} \backslash \mathbb{Q}),
$$

(3) there exists $i \in\{1, \ldots, N-1\}$ such that the following conditions hold:

a) $S_{i}$ and $S_{i+1}$ are either both orientation preserving or both orientation reversing,

b) the set

$$
\left\{\theta \in[0,2 \pi):\left[S_{i}(\gamma) \cap R_{\theta}\left(S_{i+1}(\gamma)\right)\right] \backslash\{z\} \neq \emptyset\right\}
$$

contains an open interval, where $R_{\theta}$ denotes the counterclockwise rotation by $\theta$ around the point $z:=S_{i}\left(\boldsymbol{e}_{1}\right)=S_{i+1}(\mathbf{0})$.

Then $\gamma$ is not an IFS arc.

The proof of Theorem 1.7 will show that many variants of this result are possible. Condition (3) is the only assumption that requires a priori knowledge of $\gamma$, and indeed we suspect that condition $(3)$ holds for any IFS path $(\mathcal{S}, \gamma)$ for which $\gamma$ is not a line segment.

We will give preliminary definitions in Section 2, In Section 3, we develop a key estimate, which will be used repeatedly. Section 4 gives the proof of Theorem 1.4 and of Theorem 1.5. and Section 6 gives the proof of Theorem 1.7. We conclude with a discussion of some open problems.

Acknowledgments. This work arose from the master's thesis of the first author. We wish to thank her advisor Zoltán Balogh for his kind guidance and substantial input. We also wish to thank Kari Astala and Sebastian Baader for valuable discussions. Furthermore, we extend our thanks to the two referees for carefully reading our paper and providing helpful remarks and suggestions. 


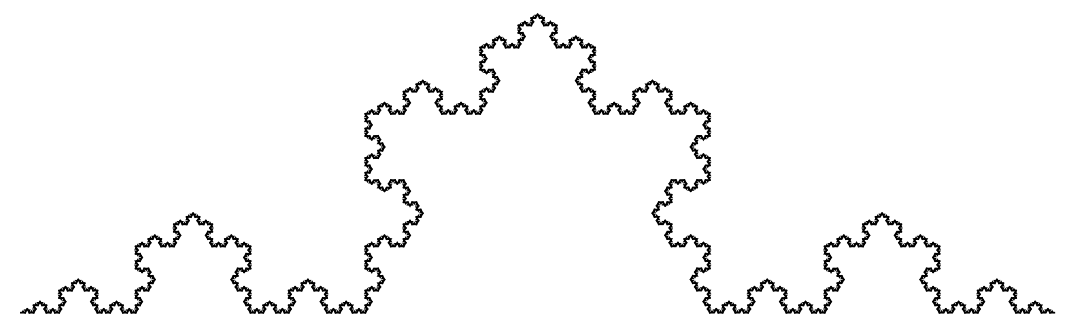

(a.)

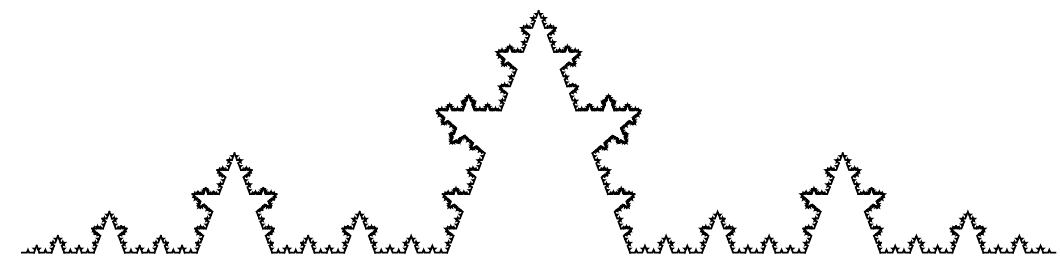

(b.)

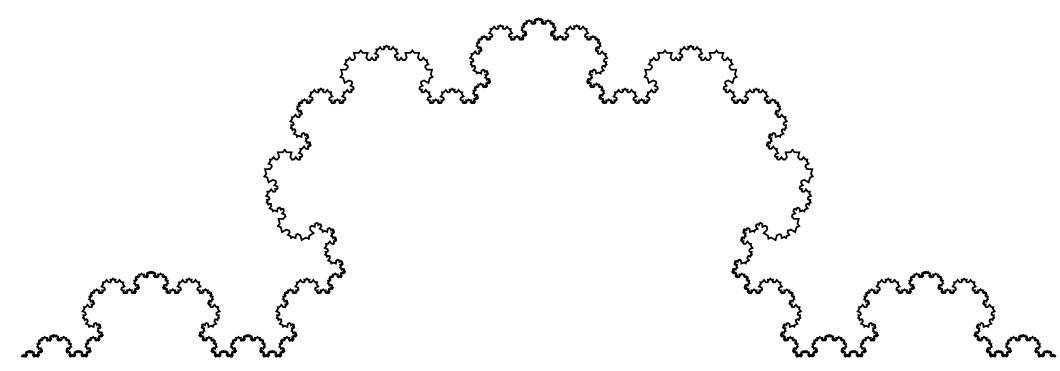

(c.)

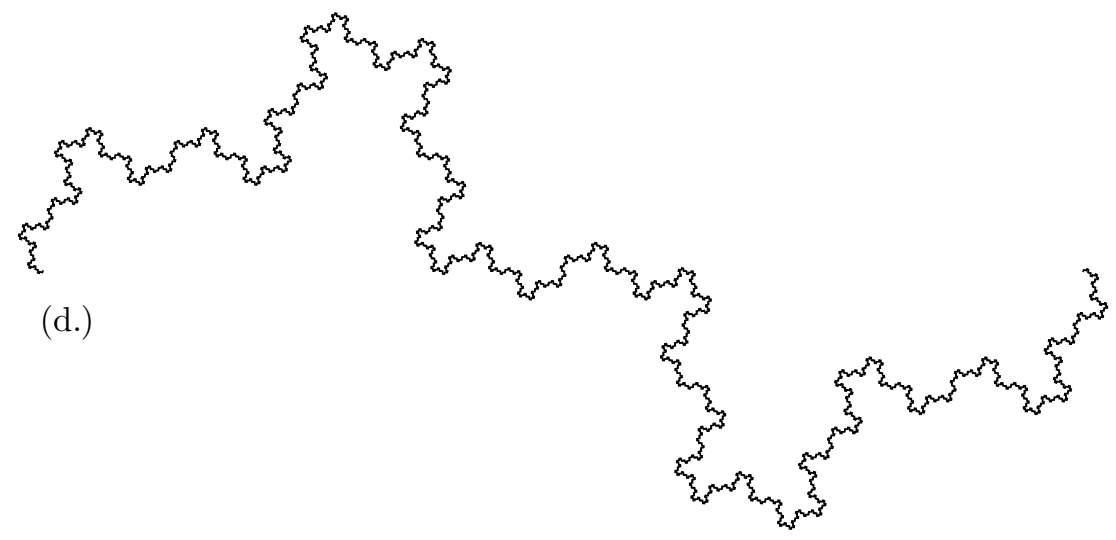

FIgURE 1. Four examples of an IFS quasiarc of similarity and Hausdorff dimension $\frac{\log (4)}{\log (3)}$. 


\section{BaCkground and Notation}

We will denote by $\mathrm{d}$ the standard distance on $\mathbb{R}^{n}$ for $n \geq 2$, by $|a-b|$ the Euclidean distance between points $a, b \in \mathbb{R}$, and by $\operatorname{diam}(E)$ the diameter of a set $E$ with respect to the metric considered on the ambient space of $E$. Furthermore, for a $(n \times n)$-matrix $A$ and a $k \in \mathbb{N}$, we will write $A^{k}$ for the matrix product $A \cdots A$ ( $k$-times), and we interpret $A^{0}=\mathrm{Id}$, where Id is the identity matrix.

A compact non-empty set $K$ is called the invariant set of an IFS $\mathcal{S}$ if $T(K)=$ $K$, where $T$ denotes the Hutchinson operator associated to $\mathcal{S}$. Each IFS $\mathcal{S}$ of contracting similarities of $\mathbb{R}^{n}$ admits a unique invariant set. Moreover, it can be constructed as a limit: Let $K_{0}$ be any non-empty compact subset of $\mathbb{R}^{n}$. Define $K_{k}$ for $k \in \mathbb{N}$ recursively by $K_{k}=T\left(K_{k-1}\right)$. Then $K$ is the limit of the sequence $\left\{K_{k}\right\}_{k \in \mathbb{N}}$ with respect to the Hausdorff distance (which is a metric on the space of non-empty compact subsets of $\mathbb{R}^{n}$ ).

Let $K$ be the invariant set of an IFS $\mathcal{S}$ of contracting similarities in $\mathbb{R}^{n}$. We call the solution $s>0$ of the equation

$$
\sum_{i=1}^{N} r_{i}^{s}=1
$$

the similarity dimension of $K$ (we assume throughout that $N \geq 2$ ). Note that for any invariant set $K$ of an IFS $\mathcal{S}$, it holds that $\operatorname{dim} K \leq s$, where $\operatorname{dim} K$ denotes the Hausdorff dimension of $K$. However, the inequality $\operatorname{dim} K \geq s$ only holds under stronger assumptions on the IFS.

2.1. Basic Notation for IFS paths. Throughout, we will assume that all IFS paths are normalized as described in the introduction. We denote the line segment between $\mathbf{0}$ and the point $\boldsymbol{e}_{1}=(1,0, \ldots, 0)$ in $\mathbb{R}^{n}$ by $I$. If $T$ is the Hutchinson operator associated to an IFS path $(\mathcal{S}, \gamma)$, then the approximation of $\gamma$ by the iterates $\left\{T^{k}(I)\right\}_{k \in \mathbb{N}}$ is an approximation by piecewise linear paths connecting $\mathbf{0}$ and $\boldsymbol{e}_{1}$.

For a sequence $\sigma=\left(\sigma_{1}, \sigma_{2}, \ldots, \sigma_{m}\right) \in\{1, \ldots, N\}^{m}$ of length $m \in \mathbb{N}$, we will write $S_{\sigma}$ for the composition of maps $S_{\sigma_{1}} \circ S_{\sigma_{2}} \circ \cdots \circ S_{\sigma_{m}}$. Analogously, we will write $r_{\sigma}$ for the product of the ratios $r_{\sigma_{1}} r_{\sigma_{2}} \ldots r_{\sigma_{m}}$. We call $p \in \gamma$ a vertex of generation $m$, if $p=S_{\sigma}(\mathbf{0})$ or $p=S_{\sigma}\left(\boldsymbol{e}_{1}\right)$ for some $\sigma \in\{1, \ldots, N\}^{m}$. We will call a set $S_{\sigma}(\gamma)$ a copy of $\gamma$ of generation $m$. Note that each vertex of generation $m$ of $\gamma$, is also a vertex of generation $m^{\prime}$ for any $m^{\prime}>m$. Similarly, each copy of $\gamma$ of generation $m^{\prime}$ is contained in a copy of $\gamma$ of generation $m$, for each $m^{\prime}>m$.

In Proposition 5.2 we will show, independently of the rest of the results in this article, that the Hutchinson parameterization of an IFS arc $(\mathcal{S}, \gamma)$ is injective, and hence that the invariant set is homeomorphic to an interval. This result will be used throughout the entire article. In particular, we will use the fact that if $(\mathcal{S}, \gamma)$ is an IFS arc, then there is a natural ordering $\leq$ on $\gamma$ that is determined by declaring that $\mathbf{0}<\boldsymbol{e}_{1}$. For a set $A \subseteq \gamma$, and a point $x \in \gamma$, we will write $x \leq A$ if $x \leq y$ for any $y \in A$. By $\gamma_{x, y}$ we denote the arc that connects $x$ to $y$ within $\gamma$, i.e., if $x \leq y$, then $\gamma_{x, y}=\{p \in \gamma: x \leq p \leq y\}$.

2.2. Quasiarcs and bounded turning. A homeomorphism $f:\left(X, d_{X}\right) \rightarrow\left(Y, d_{Y}\right)$ between metric spaces is quasisymmetric if there is a homeomorphism $\eta:[0, \infty) \rightarrow$ 
$[0, \infty)$ such that for all triples $a, b, c$ of distinct points in $X$,

$$
\frac{d_{Y}(f(a), f(b))}{d_{Y}(f(a), f(c))} \leq \eta\left(\frac{d_{X}(a, b)}{d_{X}(a, c)}\right) .
$$

Two spaces are quasisymmetrically equivalent if there is a quasisymmetric homeomorphism between them. A fundamental theorem of Tukia and Väisälä [25] states that a metric space $(X, d)$ is quasisymmetrically equivalent to the interval $[0,1]$ if and only if:

- $(X, d)$ is homeomorphic to $[0,1]$,

- $(X, d)$ is doubling, i.e., there is a constant $D \geq 1$ such that for all $r>0$, each ball of radius $r$ in $X$ can be covered by at most $D$ balls of radius $r / 2$, and

- $(X, d)$ has bounded turning, i.e., there is a constant $C \geq 1$ such that given distinct points $a, b \in X$, there is a continuum $E \subseteq X$ containing $a$ and $b$ satisfying

$$
\operatorname{diam} E \leq C d(a, b) .
$$

As mentioned in the introduction, a metric space that is quasisymmetrically equivalent to $[0,1]$ is called a quasiarc. We will prove that the invariant sets of certain IFS arcs in $\mathbb{R}^{n}$ are quasiarcs by verifying the bounded turning condition, since subsets of $\mathbb{R}^{n}$ are always doubling.

\section{Fundamental estimates}

We now provide a collection of key estimates that will be repeatedly used in the proofs of Theorem 1.4 (in order to show bounded turning) and Theorem 1.5 .

Let $(\mathcal{S}, \gamma)$ be an IFS arc in $\mathbb{R}^{n}$. Define

$$
D_{\mathcal{S}}:=\min _{z, \tilde{z}} \min \left\{\mathrm{d}(x, y): x \leq \gamma_{z, \tilde{z}} \leq y, x, y \in \gamma\right\},
$$

where the first minimum is taken over pairs of distinct generation 1 vertices $z$ and $\tilde{z}$. By Proposition 5.2, the set $\gamma$ is an arc, and so $D_{\mathcal{S}}>0$.

Fix $x, y \in \gamma$ with $x<y$. We will estimate $\mathrm{d}(x, y)$ as well as $\operatorname{diam}\left(\gamma_{x, y}\right)$ using the similarities in $\mathcal{S}$. Towards this end, let $m$ be the smallest number in $\mathbb{N}$ for which there exists a generation $m$ vertex $z$ satisfying $x \leq z \leq y$. By definition, there is a sequence $\sigma \in\{1, \ldots, N\}^{m-1}$ such that $\gamma_{x, y} \subseteq S_{\sigma}(\gamma)$.

Remark 3.1. The definition of $m$ has an (imperfect) analogue in Gromov hyperbolic geometry. Consider a tree whose vertices are labelled by finite sequences with terms in $\{1, \ldots, N\}$ and where two vertices are connected by an edge if one of the corresponding sequences extends the other. If this tree is equipped with the usual graph distance, it becomes Gromov hyperbolic, and its boundary at infinity is a Cantor set that can be labeled by infinite sequences with terms in $\{1, \ldots, N\}$ and hence maps surjectively but not injectively to $\gamma$. Given two points $\sigma$ and $\tau$ in this boundary, their Gromov product is the length of the initial sequence they share.

This analogy indicates another approach to the topic of this paper. One could study the quasisymmetry class of an IFS path $\gamma$ via quasi-isometries of its hyperbolic filling, which is a Gromov hyperbolic space whose boundary at infinity is precisely $\gamma$

We prove basic estimates in two different cases. 
Case 1. Assume that there exists another generation $m$ vertex $\tilde{z} \neq z$ with $x \leq \tilde{z} \leq$ $y$. We call the pair $(x, y)$ a case-1-pair. We may assume, without loss of generality, that $z<\tilde{z}$. This implies that $x \leq \gamma_{z, \tilde{z}} \leq y$. Applying the similarity $S_{\sigma}^{-1}$ yields $S_{\sigma}^{-1}(x) \leq S_{\sigma}^{-1}\left(\gamma_{z, \tilde{z}}\right) \leq S_{\sigma}^{-1}(y)$. Both $S_{\sigma}^{-1}(z)$ and $S_{\sigma}^{-1}(\tilde{z})$ are vertices of generation 1 , and $S_{\sigma}^{-1}\left(\gamma_{z, \tilde{z}}\right)=\gamma_{S_{\sigma}^{-1}(z), S_{\sigma}^{-1}(\tilde{z})}$. By definition, we obtain $\mathrm{d}\left(S_{\sigma}^{-1}(x), S_{\sigma}^{-1}(y)\right) \geq D_{\mathcal{S}}$ and, therefore,

$$
\mathrm{d}(x, y) \geq r_{\sigma} D_{\mathcal{S}}
$$

On the other hand, $\gamma_{x, y} \subseteq S_{\sigma}(\gamma)$, so

$$
\operatorname{diam} \gamma_{x, y} \leq \operatorname{diam}(\gamma) r_{\sigma}
$$

From (3.2) and (3.3) it follows that

$$
\operatorname{diam} \gamma_{x, y} \leq \frac{\operatorname{diam}(\gamma)}{D_{\mathcal{S}}} \mathrm{d}(x, y)
$$

Note that, in particular, this verifies the bounded turning condition for the points $x$ and $y$.

Case 2. Assume now that $z$ is the only generation $m$ vertex such that $x \leq z \leq y$. We call the pair $(x, y)$ a case-2-pair and the triple $(x, z, y)$ a case-2-triple. It follows that $x$ and $y$ are contained in adjacent copies of $\gamma$ of generation $m$. In particular, by definition, $z$ is the right endpoint of the copy of $\gamma$ of generation $m$ containing $x$ and it is the left endpoint of the copy of $\gamma$ of generation $m$ containing $y$. Thus, there exists a number $\sigma_{m} \in\{1, \ldots, N-1\}$ such that $\gamma_{x, z} \subset S_{\sigma \sigma_{m}}(\gamma)$ and $\gamma_{z, y} \subset S_{\sigma\left(\sigma_{m}+1\right)}(\gamma)$. Moreover,

$$
S_{\sigma \sigma_{m}}\left(\boldsymbol{e}_{1}\right)=z=S_{\sigma\left(\sigma_{m}+1\right)}(\mathbf{0}) .
$$

Assume for the moment that $x \neq z$. Let $k \in \mathbb{N}$ and consider all the copies of $\gamma$ of generation $m+k$ that are contained in $S_{\sigma \sigma_{m}}(\gamma)$. Note that $z$ is the right endpoint of the right-most of these copies, i.e.,

$$
z=S_{\sigma \sigma_{m}} \underbrace{N \ldots N}_{k \text { times }}\left(\boldsymbol{e}_{1}\right) .
$$

We will abuse notation here and later in similar situations by writing $\sigma \sigma_{m} N^{k}:=$ $\sigma \sigma_{m} \underbrace{N \ldots N}_{k \text { times }}$. Note that:

(i) for $k=0$, it trivially holds that $x \in S_{\sigma \sigma_{m}}(\gamma)=S_{\sigma \sigma_{m} N^{k}}(\gamma)$,

(ii) for any $k \in \mathbb{N}, S_{\sigma \sigma_{m} N^{k+1}}(\gamma) \subset S_{\sigma \sigma_{m} N^{k}}(\gamma)$, and

(iii) $\lim _{k \rightarrow \infty} \operatorname{diam}\left(S_{\sigma \sigma_{m} N^{k}}(\gamma)\right)=0$ and thus, since $x \neq z$, there will be some $k \in \mathbb{N}$ for which $x \notin S_{\sigma\left(\sigma_{m}\right) N^{k}}(\gamma)$.

By (i), (ii) and (iii), there exists $k \in \mathbb{N}$ such that

$$
x \in S_{\sigma \sigma_{m} N^{k}}(\gamma) \text { and } x \notin S_{\sigma\left(\sigma_{m}\right) N^{k+1}}(\gamma) .
$$

Set $\tilde{z}=S_{\sigma \sigma_{m} N^{k+1}}(\mathbf{0})$. Thus, $z$ and $\tilde{z}$ are distinct vertices of generation $m+k+1$ separating $x$ and $y$. Therefore, analogously to Case 1, it follows that:

$$
\mathrm{d}(x, z) \geq r_{\sigma} r_{\sigma_{m}} r_{N}^{k} D_{\mathcal{S}},
$$

as well as

$$
\operatorname{diam} \gamma_{x . z} \leq \operatorname{diam}(\gamma) r_{\sigma} r_{\sigma_{m}} r_{N}^{k}
$$


Hence,

$$
\operatorname{diam} \gamma_{x, z} \leq \frac{\operatorname{diam}(\gamma)}{D_{\mathcal{S}}} \mathrm{d}(x, z)
$$

Note that (3.9) also holds trivially if $x=z$. In either situation, if it happens that $y=z$, then (3.9) verifies the bounded turning condition for the points $x$ and $y$ with the same constant as in Case 1.

If $y \neq z$, an analogous argument shows the existence of $l \in \mathbb{N}$ such that

$$
y \in S_{\sigma\left(\sigma_{m}+1\right) 1^{l}}(\gamma) \text { and } y \notin S_{\sigma\left(\sigma_{m}+1\right) 1^{l+1}}(\gamma) .
$$

As above, it follows that

$$
\operatorname{diam} \gamma_{z, y} \leq \frac{\operatorname{diam}(\gamma)}{D_{\mathcal{S}}} \mathrm{d}(z, y)
$$

As before, (3.11) also holds trivially if $y=z$ and, in either situation, if it happens that $x=z$, then (3.9) verifies the bounded turning condition for the points $x$ and $y$ with the same constant as in Case 1 .

A consequence of these estimates is that verifying the bounded turning condition only requires estimating distances (not diameters) between the points in case-2triples.

Lemma 3.2. Let $(\mathcal{S}, \gamma)$ be an IFS arc in $\mathbb{R}^{n}$. Suppose that there is a constant $C \geq 1$ such that for all case-2-triples $(x, z, y)$,

$$
\max \{\mathrm{d}(x, z), \mathrm{d}(z, y)\} \leq C \mathrm{~d}(x, y) .
$$

Then $\gamma$ is of bounded turning with constant $\frac{2 C \operatorname{diam}(\gamma)}{D_{\mathcal{S}}}$.

Conversely, if $\gamma$ is an IFS arc of bounded turning with constant $C \geq 1$, then (3.12) holds for all points $x \leq z \leq y$ of $\gamma$.

Proof. By the arguments leading to (3.4), (3.9), and (3.11) it suffices to verify the bounded turning condition for a case-2-pair $(x, y)$ such that the corresponding case-2-triple $(x, z, y)$ satisfies $x \neq z \neq y$. By (3.9), (3.11), and (3.12), we see that

$\operatorname{diam}\left(\gamma_{x, y}\right) \leq \operatorname{diam}\left(\gamma_{x, z}\right)+\operatorname{diam}\left(\gamma_{z, y}\right) \leq \frac{\operatorname{diam}(\gamma)}{D_{\mathcal{S}}}(\mathrm{d}(x, z)+\mathrm{d}(z, y)) \leq \frac{2 C \operatorname{diam}(\gamma)}{D_{\mathcal{S}}} \mathrm{d}(x, y)$, as desired.

The proof of the converse statement is a direct consequence of the definitions.

\section{IFS ARCS WITH BOUNDED TURNING}

4.1. The proof of Theorem 1.4. A metric arc is a metric space that is homeomorphic to the interval $[0,1]$. We begin by showing that the obstacles preventing any metric arc from being of bounded turning must occur infinitesimally.

Lemma 4.1. Let $(\gamma, d)$ be an metric arc equipped with an ordering $\leq$ compatible with its topology. Let $\left(x_{i}, z_{i}, y_{i}\right)_{i \in \mathbb{N}}$ be a sequence of triples of points in $\gamma$ with $x_{i} \leq z_{i} \leq y_{i}$ and $x_{i} \neq y_{i}$ for each $i \in \mathbb{N}$. If

$$
\lim _{i \rightarrow \infty}\left(\frac{d\left(x_{i}, y_{i}\right)}{\max \left\{d\left(x_{i}, z_{i}\right), d\left(z_{i}, y_{i}\right)\right\}}\right)=0,
$$

then

$$
\lim _{i \rightarrow \infty} \max \left\{d\left(x_{i}, z_{i}\right), d\left(z_{i}, y_{i}\right)\right\}=0 .
$$


Proof. It follows from the compactness of $\gamma$ alone that (4.1) implies

$$
\lim _{i \rightarrow \infty} d\left(x_{i}, y_{i}\right)=0 \text {. }
$$

Since $\gamma$ is a metric arc, it has a parameterization by $[0,1]$ that is uniformly continuous and has uniformly continuous inverse. Hence, (4.3) implies that the diameter of the arc between $x_{i}$ and $y_{i}$ tends to 0 as well, yielding (4.2).

Remark 4.2. We will not use condition (B) in the statement of Theorem 1.4 as it is stated. Instead, we employ the following fact: For two invertible matrices $A$ and $B$, the following statements are equivalent:

(i) There exist constants $p, q \in \mathbb{N}$ such that $A^{q}=B^{p}=\mathrm{Id}$.

(ii) There exist constants $p^{\prime}, q^{\prime} \in \mathbb{N}$ such that $A^{L}=B^{L}$ for any $L, K \in \mathbb{N}$ that are multiples of $p^{\prime} q^{\prime}$.

Trivially, (i) implies (ii). The converse is an easy calculation.

Proof of Theorem 1.4. Let $(\mathcal{S}, \gamma)$ be an IFS arc in $\mathbb{R}^{n}$ satisfying the hypotheses of Theorem 1.4. We claim that if $\left(x_{i}, z_{i}, y_{i}\right)_{i \in \mathbb{N}}$ is a sequence of case-2-triples in $\gamma$ that satisfies (4.1), there exists another sequence of case-2-triples $\left(\tilde{x}_{i}, \tilde{z}_{i}, \tilde{y}_{i}\right)_{i \in \mathbb{N}}$ that satisfies (4.1) but not (4.2). Given this claim, Lemma 4.1)implies that there is no sequence of case-2-triples satisfying (4.1). Hence Lemma 3.2 yields the desired conclusion.

To this end, let $(x, y, z)$ be a case-2-triple; we may assume, without loss of generality, that $x \neq y \neq z$. Our claim will follow if we show that there exists another case-2-triple $(\tilde{x}, \tilde{z}, \tilde{y})$ satisfying the following two conditions:

$$
\begin{aligned}
& \max \{\mathrm{d}(\tilde{x}, \tilde{z}), \mathrm{d}(\tilde{z}, \tilde{y})\} \geq C, \text { where } C>0 \text { only depends on } \gamma, \\
& \frac{\mathrm{d}(x, y)}{\max \{\mathrm{d}(x, z), \mathrm{d}(z, y)\}}=\frac{\mathrm{d}(\tilde{x}, \tilde{y})}{\max \{\mathrm{d}(\tilde{x}, \tilde{z}), \mathrm{d}(\tilde{z}, \tilde{y})\}} .
\end{aligned}
$$

In case $A_{1}^{t}=A_{N}^{s}$, set $M:=s t$; in case $A_{1}^{q}=A_{N}^{p}=\mathrm{Id}$, set $M:=p q s t$, where $q, p, s, t \in \mathbb{N}$ are the numbers given in the conditions of Theorem 1.4.

As in Section 3, let $m$ be the smallest generation separating $x$ and $y$, let $\sigma \in$ $\{1, \ldots, N\}^{m-1}$ be the sequence satisfying $\gamma_{x, y} \subseteq S_{\sigma}(\gamma)$, and let $\sigma_{m} \in\{1, \ldots, N-1\}$ be the number such that

$$
S_{\sigma \sigma_{m}}\left(\boldsymbol{e}_{1}\right)=z=S_{\sigma\left(\sigma_{m}+1\right)}(\mathbf{0}) .
$$

Furthermore, let $k$ and $l$ be numbers satisfying (3.6) and (3.10), respectively; recall that these numbers locate $x$ and $y$ more precisely with respect to $z$ and the ratios $r_{1}$ and $r_{N}$.

Let $\tilde{k}$ be the largest multiple of $M$ that is no greater than $k$, and $\tilde{l}$ the largest multiple of $M$ that is no greater than $l$; note that $\widetilde{k}$ and $\widetilde{l}$ might be zero. Also, $k-\tilde{k}, l-\tilde{l} \in\{0, \ldots, M-1\}$. Without loss of generality, we assume that $\tilde{k} t \leq \tilde{l} s$; the case $\tilde{k} t \geq \tilde{l} s$ is analogous.

Set $L=\frac{\tilde{k} t}{s}$ and $K=\widetilde{k}$. Thus, $L \leq \tilde{l} \leq l$ and $K=\tilde{k} \leq k$. Therefore, $\gamma_{x, z} \subseteq$ $S_{\sigma \sigma_{m} N^{K}}(\gamma)$ and $\gamma_{z, y} \subseteq S_{\sigma\left(\sigma_{m}+1\right) 1^{L}}(\gamma)$, and we can define a mapping $\psi: \gamma_{x, y} \rightarrow \gamma$ by

$$
\psi(u)= \begin{cases}S_{\sigma_{m}} \circ\left(S_{\sigma \sigma_{m} N^{K}}\right)^{-1}(u) & u \in \gamma_{x, z}, \\ S_{\sigma_{m}+1} \circ\left(S_{\sigma\left(\sigma_{m}+1\right) 1^{L}}\right)^{-1}(u) & u \in \gamma_{z, y} .\end{cases}
$$

Set $\tilde{x}=\psi(x), \tilde{y}=\psi(y)$ and $\tilde{z}=\psi(z)$. Note that thus $(\tilde{x}, \tilde{z}, \tilde{y})$ is a case-2-triple with $\widetilde{x} \neq \widetilde{z} \neq \widetilde{y}$. Moreover: 
- On $\gamma_{x, z}$, the mapping $\psi$ is a similarity with ratio $r_{\sigma}^{-1} r_{N}^{-K}$.

- On $\gamma_{z, y}$, the mapping $\psi$ is a similarity with ratio $r_{\sigma}^{-1} r_{1}^{-L}$.

- It holds that $\gamma_{x, z} \cap \gamma_{z, y}=\{z\}$.

- By condition (A) and definition of $L$ and $L r_{1}^{-L}=r_{N}^{-K}$.

- In case that $A_{1}^{t}=A_{N}^{s}$ (see condition (B)), we have $A_{1}^{L}=A_{N}^{N}$ and therefore the angle between $x$ and $y$ at $z$ equals the angle between $\tilde{x}$ and $\tilde{y}$ at $\tilde{z}$.

- In the case when $A_{1}^{q}=A_{N}^{p}=\mathrm{Id}$ : By Remark 4.2, since $K$ and $L$ are multiples of $p q$, the angle between $x$ and $y$ at $z$ equals the angle between $\tilde{x}$ and $\tilde{y}$ at $\tilde{z}$.

From these observations, it follows that $\psi$ is a similarity of ratio $r_{\sigma}^{-1} r_{N}^{-K}$ on $\gamma_{x, y}$. So in particular, the assertion (ii) follows immediately.

On the other hand, by (3.7), it follows that $\mathrm{d}(x, z) \geq r_{\sigma} r_{\sigma_{m}} r_{N}^{k} D_{\mathcal{S}}$ and thus $\max \{\mathrm{d}(\tilde{x}, \tilde{z}), \mathrm{d}(\tilde{z}, \tilde{y})\} \geq \mathrm{d}(\tilde{x}, \tilde{z})=r_{\sigma}^{-1} r_{N}^{-K} \mathrm{~d}(x, z) \geq r_{\sigma_{m}} r_{N}^{k-K} D_{\mathcal{S}} \geq D_{\mathcal{S}}\left(\min _{1 \leq i \leq N} r_{i}\right)^{M}$, which proves (i).

We now give a list of conditions on an $\operatorname{IFS} \operatorname{arc}(\mathcal{S}, \gamma)$ in $\mathbb{R}^{2}$ that are easy to check and imply that the hypotheses of Theorem 1.4 are satisfied. Let $A_{j}: \mathbb{R}^{2} \rightarrow \mathbb{R}^{2}$ be the orthogonal transformation associated to $S_{j} \in \mathcal{S}$. Then $A_{j}: \mathbb{R}^{2} \rightarrow \mathbb{R}^{2}$ is given by the composition of a counterclockwise rotation $R_{\alpha_{j}}$ about an angle $\alpha_{j} \in[0,2 \pi)$ with a map $I_{j}$ which can be either the identity on $\mathbb{R}^{2}$ or a reflection through the $x$-axis. Thus, each similarity $S_{j}: \mathbb{R}^{2} \rightarrow \mathbb{R}^{2}$ can be written as $S_{j}(x)=$ $r_{j}\left(R_{\alpha_{j}} \circ I_{j}\right)(x)+b_{j}, x \in \mathbb{R}^{2}$, for some angle $\alpha_{j} \in[0,2 \pi)$. Equivalently, in complex coordinates, $S_{j}(z)=r_{j} e^{i \alpha_{j}} I_{j}(z)+b_{j}$, where $I_{j}$ denotes either the identity on $\mathbb{C}$ or the complex conjugation $z \mapsto \bar{z}$.

Corollary 4.3. Let $(\mathcal{S}, \gamma)$ be a normalized IFS arc in $\mathbb{R}^{2}$ with the property that

(0) there exist numbers $t, s \in \mathbb{N}$ such that $r_{1}^{t}=r_{N}^{s}$.

Assume that, in addition, one of the following conditions holds.

(1) $S_{1}$ and $S_{N}$ are orientation preserving and $\alpha_{1}, \alpha_{N} \in 2 \pi \mathbb{Q}$.

(2) $S_{1}$ and $S_{N}$ are orientation reversing.

(3) $S_{1}$ is orientation preserving, $S_{N}$ is orientation reversing, and $\alpha_{1} \in 2 \pi \mathbb{Q}$.

(4) $\alpha_{1}=\alpha_{N}$.

Then $(\mathcal{S}, \gamma)$ is a quasiarc.

Proof. Clearly, condition (0) in Corollary 4.3 equals condition (A) in Theorem 1.4. So it suffices to show that each of the conditions (1), (2), (3) and (4) of Corollary 4.3 implies condition (B) of Theorem [1.4. Assume that condition (1) holds. Then $A_{1}$ is the counterclockwise rotation $R_{\alpha_{1}}$ by the angle $\alpha_{1}$, and analogously $A_{N}=R_{\alpha_{N}}$. Moreover, there exists a number $q \in \mathbb{N}$ such that $q \alpha_{1}=q \alpha_{N}=0 \bmod 2 \pi$. Thus, $A_{1}^{q}=R_{q \alpha_{1}}=\mathrm{id}_{\mathbb{R}^{2}}$ and $A_{N}^{p}=R_{p \alpha_{N}}=\mathrm{id}_{\mathbb{R}^{2}}$, and hence (B) is satisfied. Now assume that condition (2) holds. Then each of $A_{1}$ and $A_{N}$ is a reflection of $\mathbb{R}^{2}$ over some 1-dimensional subspace of $\mathbb{R}^{2}$. Thus, $A_{1}^{2}=A_{N}^{2}=\mathrm{id}_{\mathbb{R}^{2}}$, hence (B) is satisfied. A combination of the arguments for (1) and (2) shows that (3) implies (B) as well. Finally, assume that condition (4) holds. Then $A_{1}^{2}=A_{N}^{2}$ (in case both $A_{1}$ and $A_{N}$ are orientation preserving, even $A_{1}=A_{N}$ ) and thus (B) is satisfied for $p=q=2$ (resp. $p=q=1$ ). 
It is also not difficult to come up with special circumstances under which conditions similar to the ones postulated in Corollary 4.3 work in $\mathbb{R}^{n}$. Consider, for example, the case when the similarities $S_{1}$ and $S_{N}$ of an IFS $\mathcal{S}$ in $\mathbb{R}^{n}$ are given by simple rotations: an orthogonal transformation $T$ of $\mathbb{R}^{n}$ is called a simple rotation if there exists a two-dimensional subspace $A \subset \mathbb{R}^{n}$ such that $T$ restricted to $A$ is a rotation and $T$ restricted to the orthogonal complement of $A$ is the identity.

Let us denote by $T_{A, \alpha}$ the simple rotation of $\mathbb{R}^{n}$ that is a counterclockwise rotation about the angle $\alpha \in[0,2 \pi)$ on the (oriented) two-dimensional subspace $A \subset \mathbb{R}^{n}$ and leaves the orthogonal complement of $A$ fixed. Assume that for an IFS $\mathcal{S}$ in $\mathbb{R}^{n}$ there exists a two-dimensional subspace $A \subset \mathbb{R}^{n}$ and angles $\alpha_{1}, \alpha_{N} \in[0,2 \pi)$ such that $S_{1}(x)=r_{1} T_{A, \alpha_{1}} x$ (recall that $b_{1}=0$ since $(\mathcal{S}, \gamma)$ is a normalized IFS path) and $S_{N}(x)=r_{1} T_{A, \alpha_{N}} x+b_{N}$, for all $x \in \mathbb{R}^{n}$. Then, analogously to Corollary 4.3. the following conditions imply that $\gamma$ is a quasiarc:

(1) There exist numbers $p, s \in \mathbb{N}$ and $r \in(0,1)$ such that $r_{1}=r^{s}$ and $r_{N}=r^{p}$.

(2) Either $\alpha_{1}, \alpha_{N} \in 2 \pi \mathbb{Q}$, or $\alpha_{1}=\alpha_{N}$.

4.2. The cone containment condition. We now briefly describe another, more intuitive condition guaranteeing that an $\operatorname{IFS} \operatorname{arc}(\mathcal{S}, \gamma)$ is an IFS quasiarc. While it is quite broad, it requires knowledge of the invariant set $\gamma$ that cannot be easily checked from the similarities $\mathcal{S}$ alone.

A closed cone in $\mathbb{R}^{n}$ is an isometric image of the set $\left\{v \in \mathbb{R}^{n}:\left\langle v, \boldsymbol{e}_{1}\right\rangle \geq \alpha\|v\|\right\}$ for some $0 \leq \alpha<1$. Its apex is the image of the vector $\mathbf{0}$.

Definition 4.4. We say that an IFS arc $\gamma$ given by an IFS $\mathcal{S}$ satisfies the cone containment condition if for $i \in\{2, \ldots, N-1\}$ there exist closed cones $L_{i}$ and $R_{i}$ with apex $S_{i}\left(\boldsymbol{e}_{1}\right)$ such that $L_{i} \cap R_{i}=\left\{S_{i}\left(\boldsymbol{e}_{1}\right)\right\}, S_{i}(\gamma) \subseteq L_{i}$ and $S_{i+1}(\gamma) \subseteq R_{i}$.

The following result was shown in [26]:

Theorem 4.5 (Wen and Xi, 2003). An IFS arc satisfying the cone containment condition has bounded turning.

The IFS arcs (a.), (b.), and (c.) of Figure 1 satisfy the cone containment condition. They also satisfy the conditions of Theorem 1.4 However, the IFS arc (d.) of Figure 1 satisfies the conditions of Theorem 1.4 but not the cone containment condition due to its "spiraling" behavior:

Example 4.6. Define an IFS $\mathcal{S}$ (using complex notation) by

$$
\begin{aligned}
S_{1}(z) & =\frac{1}{3} e^{i \arccos \left(\frac{3}{4}\right)} z, & S_{2}(z) & =\frac{1}{3} e^{-i \arccos \left(\frac{3}{4}\right)} z+\frac{1}{4}+i \frac{\sqrt{7}}{12}, \\
S_{3}(z) & =\frac{1}{3} e^{-i \arccos \left(\frac{3}{4}\right)} z+\frac{1}{2}, & S_{4}(z) & =\frac{1}{3} e^{i \arccos \left(\frac{3}{4}\right)} z+\frac{3}{4}-i \frac{\sqrt{7}}{12} .
\end{aligned}
$$

Note that $e^{i \arccos \left(\frac{3}{4}\right)}=\frac{3}{4}+\frac{\sqrt{7}}{4 i}$. The following scheme illustrates the mappings $S_{1}, S_{2}, S_{3}, S_{4}$ applied to the line segment $I$ :
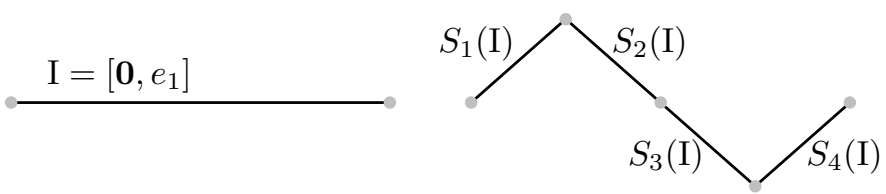
Figure 1, image (d.) illustrates the invariant set $\gamma$ of $\mathcal{S}$. Since $\alpha_{1}$ and $\alpha_{n}$ are nonzero and the similarities in $\mathcal{S}$ are orientation preserving, the arc "rotates" around each vertex. This makes it impossible for the cone containment condition to hold. However, once the non-trivial fact that $(\mathcal{S}, \gamma)$ is an IFS arc is established, it follows easily that it satisfies the conditions of Theorem 1.4. Moreover, note that the similarity dimension of this IFS arc is $\frac{\log 4}{\log 3}$, which is the same as the similarity dimension of the other IFS arcs depicted in Figure 1. Thus, by Corollary 1.6, it is bi-Lipschitz to each of the IFS paths.

Remark 4.7. The cone containment condition does not imply that the invariant set $\gamma$ is a topological arc. To see this, consider the Sierpiński gasket in $\mathbb{R}^{2}$ given by the IFS $\left\{S_{1}, S_{2}, S_{3}\right\}$ defined using complex notation by $S_{1}(z)=\frac{1}{2} e^{i \pi / 3} \bar{z}, \quad S_{2}(z)=$ $\frac{1}{2} z+\left(\frac{1}{4}+\frac{\sqrt{3}}{4} i\right), \quad S_{3}(z)=\frac{1}{2} e^{i \frac{5 \pi}{3}} \bar{z}+\left(\frac{3}{4}+\frac{\sqrt{3}}{4} i\right)$. Figure 2 illustrates the mappings $S_{1}, S_{2}, S_{3}$. Note that this example also shows that the invariant set $\gamma$ can fail to be an arc even though $T^{k}(I)$ is an arc for each $k \in \mathbb{N}$; recall that $T$ is the Hutchinson operator associated to $\mathcal{S}$ and $I$ is the interval connecting $\mathbf{0}$ and $\boldsymbol{e}_{1}$ in $\mathbb{R}^{2}$.
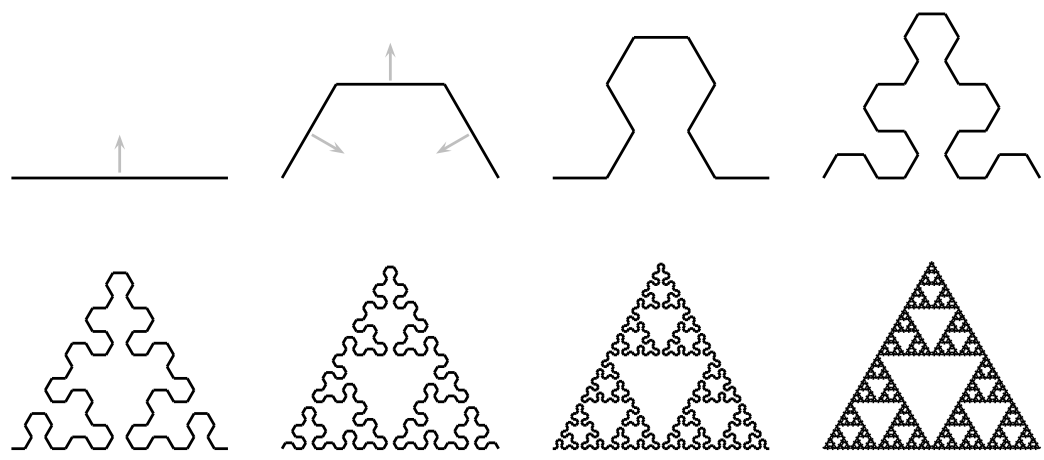

Figure 2. Sets $I$ and $T^{k}(I)$ for $k=1, \ldots, 5$. The gray arrows illustrate the orientation of the mappings $S_{1}, S_{2}$ and $S_{3}$.

\section{Parameterizations OF IFS Paths}

In this section we construct optimally Hölder continuous parameterizations of IFS paths, and show that these parameterizations are actually bi-Hölder when the IFS path is an IFS quasiarc.

5.1. The construction of structural parameterizations. In [18] Hutchinson gives a remark explaining how to construct a parameterization for an IFS path, as follows. Let $(\mathcal{S}, \gamma)$ be an IFS path. Choose a partition $\mathcal{P}$ of $[0,1]$ into $N$ pieces, i.e., fix points $0=t_{0}<t_{1}<t_{2}<\cdots<t_{N}=1$. We define a new IFS $\mathbf{s}=\left\{s_{1}, \ldots, s_{N}\right\}$ in $\mathbb{R}$ based on this partition and the original IFS $\mathcal{S}$ : For $i=1, \ldots, N$, define the mapping $s_{i}: \mathbb{R} \rightarrow \mathbb{R}$ by $s_{i}(t):=t t_{i}+(1-t) t_{i-1}$. Note that, for any $i \in\{1, \ldots, N\}$, the similarity $s_{i}$ maps the interval $[0,1]$ onto the interval $\left[t_{i-1}, t_{i}\right]$, and that the ratio of $s_{i}$ is $\left(t_{i}-t_{i-1}\right)$. The invariant set of the IFS $\mathbf{s}$ is the interval $[0,1]$. Moreover, 
$(\mathbf{s},[0,1])$ is an IFS arc in $\mathbb{R}$. In particular, for any $k \in \mathbb{N}$, we can write

$$
[0,1]=\bigcup_{\sigma \in\{1, \ldots, N\}^{k}} s_{\sigma}([0,1]) .
$$

This union is disjoint except for the vertices of generation $k$, where adjacent copies of $[0,1]$ overlap in the way described in Definition $\left[1.2\right.$. Let $\phi_{0}:[0,1] \rightarrow \mathbb{R}^{n}$ be the inclusion defined by $\phi_{0}(t):=t \boldsymbol{e}_{1}$ for all $t \in[0,1]$. Define $\phi_{k}:[0,1] \rightarrow \mathbb{R}^{n}$ by $\phi_{k}(t)=S_{\sigma} \circ \phi_{0} \circ s_{\sigma}^{-1}(t)$ for $t \in \mathbf{s}_{\sigma}([0,1])$ with $\sigma \in\{1, \ldots, N\}^{k}$. It follows that $\phi_{k}([0,1])=T^{k}(I)$, where $T$ is the Hutchinson operator associated to $\mathcal{S}$ and $I$ is the line segment connecting $\mathbf{0}$ to $\boldsymbol{e}_{1}$. Hutchinson showed in [18] that the maps $\phi_{k}$ converge uniformly to a continuous map $\phi:[0,1] \rightarrow \mathbb{R}^{n}$ with $\phi([0,1])=\gamma$.

Let $k \in \mathbb{N}$ be an integer and let $\sigma \in\{1, \ldots, N\}^{k}$. If $t \in s_{i} \circ s_{\sigma}([0,1])$, then $\phi_{k+1}(t)=S_{i} \circ \phi_{k} \circ s_{i}^{-1}(t)$, and hence it follows that

$$
S_{\sigma} \circ \phi=\phi \circ s_{\sigma},
$$

i.e., $\phi$ is a structural parameterization of $(\mathcal{S}, \gamma)$.

It turns out that there is a canonical choice of the partition $\mathcal{P}$ that optimizes the metric distortion of $\phi$ :

Definition 5.1. Let $(\mathcal{S}, \gamma)$ be an IFS path with similarity dimension $s$. The Hutchinson parameterization of $\gamma$ is the limit $\phi$ of the sequence of maps $\left\{\phi_{k}\right\}_{k \in \mathbb{N}}$ defined above where the partition $\mathcal{P}$ satisfies $t_{i}-t_{i-1}=r_{i}^{s}$ for $i \in\{1, \ldots, N\}$.

We now justify the nomenclature "IFS arc".

Proposition 5.2. Let $(\mathcal{S}, \gamma)$ be an IFS path. Then the following are equivalent:

(1) $(\mathcal{S}, \gamma)$ is an IFS arc,

(2) the Hutchinson parameterization $\phi$ of $\gamma$ is injective.

Proof of Proposition 5.2. Let $\gamma$ be an IFS arc. Assume, towards a contradiction, that the Hutchinson parameterization $\phi$ of $\gamma$ is not injective. Thus, there exist $u, v \in[0,1], u<v$, such that $\phi(u)=\phi(v)$. As in Section 3 , let $m$ be the earliest generation separating $u, v$ with respect to $(\mathbf{s},[0,1])$, and $q$ a vertex of generation $m$ such that $u<q<v$. Thus, there exist $\sigma \in\{1, \ldots, N\}^{m-1}$ and $i<j \in\{1, \ldots, N\}$ such that $u \in s_{\sigma} s_{i}([0,1])$ and $v \in s_{\sigma} s_{j}([0,1])$ with $u \neq s_{\sigma} s_{i}(1)$ and $u \neq s_{\sigma} s_{j}(0)$.

Then, since $\phi(u)=\phi(v)$, the set $S_{\sigma} S_{i}(\gamma) \cap S_{\sigma} S_{j}(\gamma)$ contains a point which is not $S_{\sigma} S_{i}\left(\boldsymbol{e}_{1}\right)$ or $S_{\sigma} S_{j}(\mathbf{0})$. Applying $S_{\sigma}^{-1}$ yields a contradiction.

That $(\mathcal{S}, \gamma)$ is an IFS arc if $\phi$ is injective follows quickly from the definitions.

5.2. Hölder continuity of the Hutchinson parameterization. The following result can be found in [15] and [1], but we include the proof for completeness as it is a simple consequence of the basic estimates of Section 3 .

Theorem 5.3. Let $(\mathcal{S}, \gamma)$ be an IFS path with similarity dimension s. Then Hutchinson parameterization of $\gamma$ is $\frac{1}{s}$-Hölder.

Proof. Let $u<v$ be distinct points of $[0,1]$. We will employ the estimates of Section 3 with respect to the IFS path $\mathbf{s}$ defined above. Namely,

(1) let $m$ be the smallest generation separating $u$ and $v$,

(2) let $q$ be a generation $m$ vertex satisfying $u \leq q \leq v$,

(3) let $\sigma \in\{1, \ldots, N\}^{m-1}$ be the sequence satisfying $[u, v] \subseteq s_{\sigma}([0,1])$, 
If $(u, v)$ is a case-1-pair, then (3) above, (5.1), and the fundamental estimate (3.2) imply that

$$
\mathrm{d}(\phi(u), \phi(v)) \leq \operatorname{diam}(\gamma) r_{\sigma} \leq \frac{\operatorname{diam}(\gamma)}{D_{\mathbf{s}}}|u-v|^{\frac{1}{s}} .
$$

Now, assume that $(u, v)$ is a case-2-pair and thus $(u, q, v)$ is a case-2-triple. We may assume, without loss of generality, that

$$
\mathrm{d}(\phi(u), \phi(v)) \leq 2 \mathrm{~d}(\phi(u), \phi(q)) .
$$

As in Section 3 ,

- let $\sigma_{m} \in\{1, \ldots, N-1\}$ be the number such that $s_{\sigma \sigma_{m}}(1)=z=s_{\sigma\left(\sigma_{m}+1\right)}(0)$,

- let $k \in \mathbb{N}$ be the number satisfying $u \in s_{\sigma \sigma_{m} N^{k}}([0,1])$ and $u \notin s_{\sigma \sigma_{m} N^{k+1}}([0,1])$. Then $u, q \in s_{\sigma, \sigma_{m}, N^{k}}([0,1])$, and so $\phi(u), \phi(q) \in S_{\sigma, \sigma_{m}, N^{k}}(\gamma)$. Thus, by applying (5.2),

$$
\mathrm{d}(\phi(u), \phi(v)) \leq 2 \operatorname{diam}(\gamma) r_{\sigma} r_{\sigma_{m}} r_{N}^{k} .
$$

On the other hand, using (3.7) and the fact that $u \leq q \leq v$ are points in $[0,1]$, we see that

$$
|u-v| \geq|u-q| \geq D_{\mathbf{s}}\left(r_{\sigma} r_{\sigma_{m}} r_{N}^{k}\right)^{s},
$$

and thus $\mathrm{d}(\phi(u), \phi(v)) \leq 2 \frac{\operatorname{diam}(\gamma)}{D_{\mathbf{s}}}|u-v|^{\frac{1}{s}}$. This completes the proof.

5.3. Bi-Hölder continuity of the Hutchinson parameterization. We now use the estimates of Section 3 to prove Theorem 1.5 .

Proof of Theorem 1.5. Let $(\mathcal{S}, \gamma)$ be an IFS quasiarc and let $s$ denote its similarity dimension. Let $u<v$ be distinct points of $[0,1]$. Given Theorem [5.3, in order to establish the $\frac{1}{s}$-bi-Hölder continuity of the Hutchinson parameterization $\phi$, we must only produce an appropriate lower bound for $\mathrm{d}(\phi(u), \phi(v))$.

Let $(\mathbf{s},[0,1])$ be the IFS arc used in the construction of $\phi$ (see Section [5.1), and let $m, q$, and $\sigma$ be as defined in (1)-(3) of the proof of Theorem 5.3. First, assume that $(u, v)$ is a case-1-pair with respect to the IFS arc $(\mathbf{s},[0,1])$. Then $(\phi(u), \phi(v))$ is a case-1-pair with respect to the IFS $\operatorname{arc}(\mathcal{S}, \gamma)$ and so by (3.2) applied to $(\mathcal{S}, \gamma)$ and (3.3) applied to $(\mathbf{s},[0,1])$,

$$
\mathrm{d}(\phi(u), \phi(v)) \geq D_{\mathcal{S}}|u-v|^{\frac{1}{s}} .
$$

Now assume that $(u, q, v)$ is a case-2-triple with respect to the $(\mathbf{s},[0,1])$. Then $(\phi(u), \phi(q), \phi(v))$ is a case-2-triple with respect to $(\mathcal{S}, \gamma)$. Without loss of generality, we may assume that $|u-q| \geq|q-v|$. Let $C$ denote the bounded turning constant of $\gamma$. Then by Lemma 3.2

$$
\mathrm{d}(\phi(u), \phi(v)) \geq \frac{\mathrm{d}(\phi(u), \phi(q))}{C} .
$$

Let $\sigma_{m} \in\{1, \ldots, N-1\}$ and let $k \in \mathbb{N}$ be such that (3.6) holds for the triple $(\phi(u), \phi(q), \phi(v))$. Then (3.7) for this triple implies

$$
\mathrm{d}(\phi(u), \phi(q)) \geq D_{\mathcal{S}} r_{\sigma} r_{\sigma_{m}} r_{N}^{k} .
$$

By (5.1), $\sigma_{m}$ and $k$ would remain unchanged had we chosen them based on the triple $(u, q, v)$ with respect to the IFS $(\mathbf{s},[0,1])$, and so (3.8) applied to the IFS $\mathbf{s}$ yields

$$
|u-q| \leq\left(r_{\sigma} r_{\sigma_{m}} r_{N}^{k}\right)^{s}
$$


As we have assumed $|u-q| \geq|q-v|$, we conclude that

$$
\mathrm{d}(\phi(u), \phi(v)) \geq \frac{D_{\mathcal{S}}}{2 C}|u-v|^{\frac{1}{s}} .
$$

Conversely, if the Hutchinson parameterization $\phi$ is $\frac{1}{s}$-bi-Hölder continuous, then it is also quasisymmetric. The bounded turning condition is preserved under quasisymmetries [25], and so the fact that $[0,1]$ has bounded turning implies that $\gamma$ has bounded turning. Thus, $\gamma$ is a quasiarc.

In fact, if $(\mathcal{S}, \gamma)$ is an IFS quasiarc, then the Hutchinson parameterization is the $s$-dimensional arclength parameterization of $\gamma$.

Corollary 5.4. Let $(\mathcal{S}, \gamma)$ be an IFS quasiarc with similarity dimension s. Then the Hutchinson parameterization $\phi$ satisfies

$$
\mathscr{H}^{s}(\phi[u, v])=\mathscr{H}^{s}(\gamma)|u-v|,
$$

for all $u, v \in[0,1]$.

Proof. For the moment, fix $\sigma \in\{1, \ldots, N\}^{k}$ and $k \in \mathbb{N}$. Since $\phi$ is $\frac{1}{s}$-bi-Hölder continuous, it holds that $0<\mathscr{H}^{s}(\gamma)<\infty$. Hence, $\mathscr{H}^{s}\left(S_{\sigma}(\gamma)\right)=\mathscr{H}^{s}(\gamma) r_{\sigma}^{s}$. On the other hand, $r_{\sigma}^{s}=\mathscr{H}^{1}\left(s_{\sigma}([0,1])\right)$. Thus, since $\phi\left(s_{\sigma}([0,1])\right)=S_{\sigma}(\gamma)$, it follows that

$$
\mathscr{H}^{s}\left(\phi\left(s_{\sigma}([0,1])\right)\right)=\mathscr{H}^{s}(\gamma) \mathscr{H}^{1}\left(s_{\sigma}([0,1])\right) .
$$

Let $u, v \in[0,1]$. Define the sequence of collections of intervals $\left(M_{k}\right)_{k \in \mathbb{N}}$ as follows:

$M_{1}=\left\{s_{\sigma}([0,1]) \subseteq[u, v]: \sigma \in\{1, \ldots, N\}\right\}$,

$M_{k}=\left\{s_{\sigma}([0,1]) \subseteq[u, v]: \sigma \in\{1, \ldots, N\}^{k}, s_{\sigma}([0,1]) \nsubseteq I\right.$ for any $I \in M_{j}$ with $\left.j<k\right\}$.

Then

$$
(u, v) \subseteq \bigcup_{k \in \mathbb{N}} \bigcup_{I \in M_{k}} I \subseteq[u, v] .
$$

Moreover, any two intervals in this union intersect in at most one point. Hence,

$$
\phi((u, v)) \subseteq \bigcup_{k \in \mathbb{N}} \bigcup_{I \in M_{k}} \phi(I) \subseteq \phi([u, v]) .
$$

Since $(\mathcal{S}, \gamma)$ is an IFS arc, Proposition 5.2 implies that any two of the terms above intersect in at most one point as well. Since points have zero $s$-dimensional Hausdorff measure, (5.3) implies that

$$
\mathscr{H}^{s}(\phi[u, v])=\sum_{k \in \mathbb{N}} \sum_{I \in M_{k}} \mathscr{H}^{s}(\phi(I))=\sum_{k \in \mathbb{N}} \sum_{I \in M_{k}} \mathscr{H}^{s}(\gamma) \mathscr{H}^{1}(I)=\mathscr{H}^{s}(\gamma) \mathscr{H}^{1}([u, v]),
$$

as desired.

To put Corollary [5.4 in context, it can be gleaned from [20, [10], and [11, or slightly more directly from [14], that any Ahlfors $s$-regular quasiarc has an $s$ dimensional arclength parameterization by $[0,1]$ that is $\frac{1}{s}$-bi-Hölder continuous; the novelty of Corollary 5.4 is that for IFS quasiarcs, this parameterization coincides with the Hutchinson parameterization. 
5.4. An Example. Define the IFS $\mathcal{S}(a, b)$ depending on two parameters $a, b \in$ $\left(0, \frac{1}{2}\right)$ (using complex notation) by

$$
\begin{array}{ll}
S_{1}(z)=a z, & S_{2}(z)=b e^{i \arccos \left(b^{-1}\left(\frac{1}{2}-a\right)\right)} z+a, \\
S_{3}(z)=b e^{-i \arccos \left(b^{-1}\left(\frac{1}{2}-a\right)\right)} z+\frac{1}{2}+i h, & S_{4}(z)=a z+(1-a),
\end{array}
$$

where $h=\sqrt{b^{2}-\left(\frac{1}{2}-a\right)^{2}}$. .

The following figure illustrates the mappings $S_{1}, S_{2}, S_{3}, S_{4}$ applied to the segment $I$.
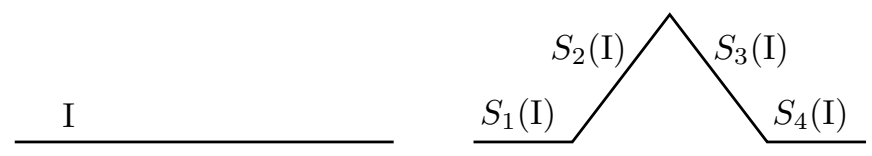

One can easily show that for any parameters $a, b \in\left(0, \frac{1}{2}\right)$, the invariant set $\gamma(a, b)$ of $\mathcal{S}(a, b)$ is a quasiarc (either by applying Theorem[1.4, or using the cone containment condition). The similarity dimension (and hence Hausdorff dimension) of $\gamma$ is the unique solution $s$ of $2 a^{s}+2 b^{s}=1$. Thus, for each $s \in(0,2)$, such examples provide a one-parameter family of bi-Lipshitz equivalent IFS quasiarcs of dimension $s$. Figure 11 images (a.), (b.) and (c.), are members of this family where $s=\frac{\log (4)}{\log (3)}$; image (d.) is also an IFS quasiarc of this dimension and hence bi-Lipschitz equivalent to the other arcs displayed, but arises from the IFS described in Example 4.6.

5.5. Approximate self-similarity and bounded turning. In many contexts, the space $(X, d)$ of interest is not known to be the invariant set of a family of contracting similarities, but rather only known to possess a more flexible form of self-similarity:

- scaled bi-Lipschitz copies of any small-scale piece of $(X, d)$ appear at the top scale, and

- a scaled bi-Lipschitz copy of $(X, d)$ appears at every scale and location.

A metric space satisfying the first condition above was called quasi-self-similar by Mclauglin [20] and approximate self-similar in [7]; a metric space homeomorphic to a circle that satisfies both conditions was called a quasicircle by Falconer-Marsh 11. Carrasco Piaggio has shown that an approximately self-similar locally connected metric space has bounded turning [8], which implies that a "Falconer-Marsh quasicircle" is indeed the quasisymmetric image of $\mathbb{S}^{1}$ (the converse of this statement is false).

Taken together, the papers of McLaughlin [20, Falconer [10, and FalconerMarsh [1] imply the following theorem:

Theorem 5.5. A Falconer-Marsh quasicircle possesses a bi-Hölder parameterization by $\mathbb{S}^{1}$.

Thus, an alternate approach to Theorem [1.5] is to show that the invariant set of an IFS quasiarc satisfies the two conditions given above and then proves a version of Theorem 5.5 for arcs. This is much less direct than the approach we have taken; however, it turns out to be logically equivalent, as the following result shows:

Theorem 5.6. The invariant set of an $\operatorname{IFS}$ arc $(\mathcal{S}, \gamma)$ has bounded turning if and only if the following two conditions hold: 
(1) There exist constants $r_{0}>0$ and $C_{1}, C_{2}>0$ such that for every open set $U \subseteq \mathbb{R}^{n}$ satisfying $U \cap \gamma \neq \emptyset$ and $0<\operatorname{diam}(U \cap \gamma)<r_{0}$, there exists a mapping $\varphi: U \cap \gamma \rightarrow \gamma$ such that

$$
\frac{C_{1} \mathrm{~d}(a, b)}{\operatorname{diam}(U \cap \gamma)} \leq \mathrm{d}(\varphi(a), \varphi(b)) \leq \frac{C_{2} \mathrm{~d}(a, b)}{\operatorname{diam}(U \cap \gamma)}
$$

for all $a, b \in U \cap \gamma$.

(2) There exist constants $r_{1}>0$ and $C_{3}>0$ such that for any $x \in \gamma$ and radius $0<r<r_{1}$, there is a mapping $\psi: \Gamma \rightarrow B(x, r) \cap \Gamma$ satisfying

$$
\mathrm{d}(\psi(a), \psi(b)) \geq C_{3} r \mathrm{~d}(a, b)
$$

for all $a, b \in \gamma$.

Proof. That bounded turning implies the two conditions given in the statement follows immediately from Theorem 1.5, as they are invariant under bi-Hölder changes of metric and are clearly satisfied by the interval $[0,1]$.

Now suppose that $(\mathcal{S}, \gamma)$ is an IFS arc such that condition (1) of Theorem 5.6 holds. That $\gamma$ has bounded turning follows from the work of Piaggio Carrasco [8], but we can give a short, self-contained proof in this special setting.

Assume, towards a contradiction, that $\gamma$ is not of bounded turning. Then there is a sequence of pairs of points $\left\{\left(x_{i}, y_{i}\right)\right\}_{i \in \mathbb{N}}$ of $\gamma$ such that $x_{i}<y_{i}$, and

$$
\lim _{i \rightarrow \infty} \frac{\mathrm{d}\left(x_{i}, y_{i}\right)}{\operatorname{diam}\left(\gamma_{x_{i}, y_{i}}\right)}=0 \text {. }
$$

Define $\rho_{i}:=\operatorname{diam}\left(\gamma_{x_{i}, y_{i}}\right)$. As $\gamma$ is an arc, it must be the case that $\lim _{i \rightarrow \infty} \rho_{i}=0$. Hence, we may assume, without loss of generality, that $2 \rho_{i}<r_{0}$ for each $i \in \mathbb{N}$.

Fix $i \in \mathbb{N}$. Let $\varphi_{i}: B\left(x_{i}, 2 \rho_{i}\right) \cap \gamma \rightarrow \gamma$ be the mapping provided by condition (1) with $U=B\left(x_{i}, 2 \rho_{i}\right)$. Since the mapping $\varphi_{i}$ is a topological embedding, $\varphi_{i}\left(\gamma_{x_{i}, y_{i}}\right)=$ $\gamma_{\varphi_{i}\left(x_{i}\right), \varphi\left(y_{i}\right)}$. Hence, the estimates of condition (1) yield

$$
\operatorname{diam}\left(\gamma_{\varphi_{i}\left(x_{i}\right), \varphi_{i}\left(y_{i}\right)}\right) \geq \frac{C_{1} \operatorname{diam}\left(\gamma_{x_{i}, y_{i}}\right)}{\operatorname{diam}(U \cap \gamma)}
$$

as well as

$$
\lim _{i \rightarrow \infty} \frac{\mathrm{d}\left(\varphi_{i}\left(x_{i}\right), \varphi_{i}\left(y_{i}\right)\right)}{\operatorname{diam}\left(\gamma_{\varphi_{i}\left(x_{i}\right), \varphi_{i}\left(y_{i}\right)}\right)} \leq \lim _{i \rightarrow \infty} \frac{C_{2} \mathrm{~d}\left(x_{i}, y_{i}\right)}{C_{1} \operatorname{diam}\left(\gamma_{x_{i}, y_{i}}\right)}=0 .
$$

However, $\operatorname{diam}(U \cap \gamma) \leq 2 \rho_{i}=2 \operatorname{diam}\left(\gamma_{x_{i}, y_{i}}\right)$ and thus (5.4) implies that $\operatorname{diam}\left(\gamma_{\varphi_{i}\left(x_{i}\right), \varphi_{i}\left(y_{i}\right)}\right) \geq \frac{C_{1}}{2}$. Since $\gamma$ is an arc, this contradicts (5.5).

Remark 5.7. As the proof shows, condition (1) alone implies that an IFS arc is an IFS quasiarc.

\section{IFS PATHS WITH NON-INJECTIVE HUTCHINSON PARAMETRIZATION}

The goal of this section is to prove Theorem 1.7 and to give several sets of sufficient conditions in order for Theorem 1.7 to hold.

Proof of Theorem 1.7. Recall that $z:=S_{i}\left(\boldsymbol{e}_{1}\right)=S_{i+1}(\mathbf{0})$. By condition (3.b) of the statement of Theorem 1.7, the set

$$
T:=\left\{\theta \in[0,2 \pi):\left[S_{i}(\gamma) \cap R_{\theta}\left(S_{i+1}(\gamma)\right)\right] \backslash\{z\} \neq \emptyset\right\}
$$

contains an open interval $I$. 
Since $t \alpha_{1}-s \alpha_{N} \in 2 \pi(\mathbb{R} \backslash \mathbb{Q})$, there exists $m \in \mathbb{N}$ such that $\theta_{0}:=m\left(t \alpha_{1}-s \alpha_{N}\right) \in$ $I \subseteq T$. Thus, the sets $S_{i}(\gamma)$ and $R_{\theta_{0}}\left(S_{i+1}(\gamma)\right)$ intersect in a point $\tilde{z} \neq z$. In particular, this implies that $S_{i}(\gamma) \cup R_{\theta_{0}}\left(S_{i+1}(\gamma)\right)$ contains a homeomorphic copy of $\mathbb{S}^{1}$

Regarding condition (3.a), assume that both $S_{i}$ and $S_{i+1}$ are orientation preserving; the case that they are both orientation reversing is analogous. Now consider the sets $S_{i} \circ S_{N}^{m s}(\gamma), S_{i+1} \circ S_{1}^{m t}(\gamma) \subset \gamma$. By condition (1), the similarity $S_{N}$ rotates $\gamma$ by an angle of $\alpha_{N}$ around the point $\boldsymbol{e}_{1}$, while $S_{1}$ rotates $\gamma$ by an angle of $\alpha_{1}$ around $\mathbf{0 .}$.

Therefore, the angle between $S_{i} \circ S_{N}^{m s}(\gamma)$ and $S_{i+1} \circ S_{1}^{m t}(\gamma)$ at $z$ is the angle between $S_{i}(\gamma)$ and $S_{i+1}(\gamma)$ at $z$ plus $m\left(t \alpha_{1}-s \alpha_{n}\right)$. By condition $(2), r_{1}^{m t}=r_{N}^{m s}$, and hence $\left(S_{i} \circ S_{N}^{m s}(\gamma)\right) \cup\left(S_{i+1} \circ S_{1}^{m t}(\gamma)\right)$ is the image of $S_{i}(\gamma) \cup R_{\theta_{0}}\left(S_{i+1}(\gamma)\right)$ under a similarity. Thus, $\gamma$ contains a homeomorphic copy of $\mathbb{S}^{1}$, and hence $\gamma$ can not be the homeomorphic image of an interval.

We now indicate one situation in which the hypotheses of Theorem 1.7 hold. While somewhat simpler, it still requires some a priori knowledge of the IFS path.

Corollary 6.1. Let $(\mathcal{S}, \gamma)$ be a normalized IFS path in $\mathbb{R}^{2}$ that satisfies conditions (1) and (2) of Theorem 1.7. Assume that:

(3*) (a) either both $S_{i}$ and $S_{i+1}$ are orientation preserving, or both are orientation reversing;

(b) there is no rotation around the point $S_{i}\left(\boldsymbol{e}_{1}\right)=S_{i+1}(\mathbf{0})$ that maps $S_{i}(\gamma)$ into $S_{i+1}(\gamma)$, or vice versa.

Then condition (3) of Theorem 1.7 holds and so $\gamma$ is not an IFS arc.

In order to prove this, we will need a topological lemma. Consider the punctured disk

$$
A:=\{z \in \mathbb{C}: 0<|z|<1\},
$$

and its closure $\bar{A}$, which coincides with the closed unit disk. For each $\theta \in \mathbb{S}^{1} \subseteq \mathbb{C}$, define a mapping $R_{\theta}: \mathbb{C} \rightarrow \mathbb{C}$ by $R_{\theta}(z)=\theta z$.

Lemma 6.2. Let $\alpha:[0,1] \rightarrow \bar{A}$ be an injective path. Consider another injective path $\beta:[0,1] \rightarrow \bar{A}$ such that

- $\beta(0)=0$,

- $\beta(s) \in A$ for all $s \in(0,1)$,

- $|\beta(1)|=1$,

- $\operatorname{im} \alpha \cap \operatorname{im} \beta \subseteq\{0\}$ (where $\operatorname{im} \alpha$ and $\operatorname{im} \beta$ denote the images of $\alpha$ and $\beta$, respectively).

Furthermore, assume that there does not exist $\theta \in \mathbb{S}^{1}$ such that

$$
\operatorname{im}\left(R_{\theta} \circ \alpha\right) \subseteq \operatorname{im}(\beta) .
$$

Then the set

$$
T:=\left\{\theta \in \mathbb{S}^{1}:\left(\operatorname{im}\left(R_{\theta} \circ \alpha\right) \cap \operatorname{im}(\beta)\right) \backslash\{0\} \neq \emptyset\right\}
$$

contains an interval.

Proof of Lemma 6.2. Consider the strip $\widetilde{A}=(0,1] \times \mathbb{R}$ as the universal cover of the punctured closed disk $A^{\prime}=\bar{A} \backslash\{0\}$ with the associated projection $\pi: \widetilde{A} \rightarrow A^{\prime}$ 
defined by $\pi(r, t)=r e^{i t}$. Let $\widetilde{\alpha}$ and $\widetilde{\beta}$ be lifts of $\left.\alpha\right|_{(0,1]}$ and $\left.\beta\right|_{(0,1]}$ to $\widetilde{A}$, respectively; then $\widetilde{\alpha}$ and $\widetilde{\beta}$ have disjoint images.

For $t \in \mathbb{R}$, define $\widetilde{\alpha}_{t}$ by $\widetilde{\alpha}+(0, t)$. Then the image of $\pi\left(\widetilde{\alpha}_{t}\right)$ coincides with the image of $R_{\theta} \circ \alpha$ for $\theta=e^{i t}$. Thus, it suffices to show that the set

$$
\widetilde{T}=\left\{t \in \mathbb{R}:\left(\operatorname{im}\left(\widetilde{\alpha}_{t}\right) \cap \operatorname{im}(\widetilde{\beta})\right) \neq \emptyset\right\}
$$

contains an interval.

By assumption, there is no $t \in \mathbb{R} \operatorname{such}$ that $\operatorname{im}\left(\widetilde{\alpha}_{t}\right) \subseteq \operatorname{im}(\widetilde{\beta})$. Note that $\widetilde{A} \backslash \widetilde{\beta}$ has exactly two components, which we denote by $L$ and $U$. Define

$$
t_{0}:=\sup \left\{t \in \mathbb{R}: \operatorname{im} \widetilde{\alpha}_{t} \subseteq L\right\} \text { and } t_{1}:=\inf \left\{t \in \mathbb{R}: \operatorname{im} \widetilde{\alpha}_{t} \subseteq U\right\} .
$$

We may assume, without loss of generality, that $0 \leq t_{0}<\infty$ and that $t_{0} \leq t_{1}<\infty$. Suppose that $t_{0}<t_{1}$; then for each $t \in\left(t_{0}, t_{1}\right)$, the set $\operatorname{im}\left(\widetilde{\alpha}_{t}\right)$ is neither contained in $L$ nor $U$, and so $t \in \widetilde{T}$. This shows that $\widetilde{T}$ contains an interval.

Now suppose that $t_{0}=t_{1}$. Then, for any $\epsilon>0$, it holds that $\operatorname{im}\left(\widetilde{\alpha}_{t_{0}-\epsilon}\right) \subseteq L$ while $\operatorname{im}\left(\widetilde{\alpha}_{t_{0}+\epsilon}\right) \subseteq U$. This implies that $\operatorname{im}\left(\widetilde{\alpha}_{t_{0}}\right)$ is contained in $\operatorname{im}(\widetilde{\beta})$, a contradiction.

Proof of Corollary 6.1. Let $(\mathcal{S}, \gamma)$ be a normalized IFS path in $\mathbb{R}^{2}$ that satisfies conditions (1) and (2) of Theorem 1.7.

By assumption, we may find an index $i \in\{1, \ldots, N-1\}$ so that both $S_{i}$ and $S_{i+1}$ are orientation preserving or both are orientation reversing and, moreover, that there is no rotation around $z:=S_{i}\left(\boldsymbol{e}_{1}\right)=S_{i+1}(\mathbf{0})$ that maps $S_{i}(\gamma)$ onto a subset of $S_{i+1}(\gamma)$ or vice-versa. We assume that

$$
\max \left\{\mathrm{d}(x, z): x \in S_{i}(\gamma)\right\} \leq R:=\max \left\{\mathrm{d}(z, y): y \in S_{i+1}(\gamma)\right\},
$$

as a similar argument is valid if this is not the case. Furthermore, define

$$
t_{0}:=\psi^{-1}\left(S_{i}(\mathbf{0})\right), t_{1}:=\psi^{-1}(z) \text { and } t_{2}:=\min \left\{t \in \psi^{-1}\left(S_{i+1}(\gamma)\right): \mathrm{d}(z, \psi(t))=R\right\} \text {. }
$$

Consider the punctured disk $A:=\left\{p \in \mathbb{R}^{2}: 0<\mathrm{d}(z, p)<R\right\}$ and the injective paths $\alpha, \beta$ defined by $\alpha:=\left.\psi\right|_{\left[t_{0}, t_{1}\right]}$ and $\beta=\left.\psi\right|_{\left[t_{1}, t_{2}\right]}$. Note that thus $\beta\left(t_{1}\right)=z, \beta(s) \in A$ for all $s \in\left(t_{1}, t_{2}\right),\left|\beta\left(t_{2}\right)\right|=R$ and $\operatorname{im} \alpha \cap \operatorname{im} \beta=\{z\}$. Moreover, as we have stated above, there does not exist an angle $\theta \in \mathbb{S}^{1}$ such that $\operatorname{im}\left(R_{\theta} \circ \alpha\right) \subseteq \operatorname{im}(\beta)$. Thus, after shifting, scaling, and reparameterizing, Lemma 6.2 shows that the set

$$
T:=\left\{\theta \in \mathbb{S}^{1}:\left(\operatorname{im}\left(R_{\theta} \circ \alpha\right) \cap \operatorname{im}(\beta)\right) \backslash\{z\} \neq \emptyset\right\}
$$

contains an interval $I$.

Remark 6.3. Theorem 1.7 still holds under either one of the following two sets of slightly different assumptions: Let $(\mathcal{S}, \gamma)$ be a normalized IFS path in $\mathbb{R}^{2}$ such that:

(1') $S_{1}$ is orientation preserving, $S_{N}$ is orientation reversing,

(2') there exists a number $t \in \mathbb{N}$ such that $r_{1}^{t}=r_{N}^{2}$, and $t \alpha_{1} \in 2 \pi(\mathbb{R} \backslash \mathbb{Q})$,

(3') there exists $i \in\{1, \ldots, N-1\}$ such that the following conditions hold:

a) $S_{i}$ and $S_{i+1}$ are either both orientation preserving or both orientation reversing,

b) the set $\left\{\theta \in[0,2 \pi):\left[S_{i}(\gamma) \cap R_{\theta}\left(S_{i+1}(\gamma)\right)\right] \backslash\{z\} \neq \emptyset\right\}$ contains an open interval

and

(1") $S_{1}$ and $S_{N}$ are orientation preserving,

(2") there exist numbers $t, s \in \mathbb{N}$ such that $r_{1}^{t}=r_{N}^{s}$, and $\left(t \alpha_{1}+s \alpha_{N}\right) \in 2 \pi(\mathbb{R} \backslash \mathbb{Q})$,

(3") there exists $i \in\{1, \ldots, N-1\}$ such that the following conditions hold: 
a) $S_{i}$ is orientation preserving and $S_{i+1}$ is orientation reversing (or vice versa),

b) the set $\left\{\theta \in[0,2 \pi):\left[S_{i}(\gamma) \cap R_{\theta}\left(S_{i+1}(\gamma)\right)\right] \backslash\{z\} \neq \emptyset\right\}$ contains an open interval.

The proofs are analogous to the proof of Theorem 1.7 by just carefully reconsidering with what angle $S_{N}$ and $S_{1}$ rotate $\gamma$ around 0 and $\boldsymbol{e}_{1}$, respectively, and taking into account how an orientation reversing similarity transforms angles. Also, one can deduce corollaries analogous to Corollary 6.1 from the above variants of Theorem 1.7

\section{OPEN QUESTIONS AND FURTHER DIRECTIONS FOR RESEARCH}

The obvious task left uncompleted by this work is an optimal version of Theorem 1.4.

Problem 7.1. Give necessary and sufficient conditions for an IFS arc $(\mathcal{S}, \gamma)$ to be an IFS quasiarc in terms of the similarities in $\mathcal{S}$ alone.

It would be particularly interesting if there was a simple characterization of IFS quasiarcs, rather than an exhaustive list of cases. A similar question can be posed regarding necessary and sufficient conditions for an IFS path to be an IFS arc; this question is likely very difficult. More approachable is the question of sharpness of Theorem 1.7

Problem 7.2. Does Theorem 1.7 hold without the assumption of condition (3)?

The bounded turning condition makes sense for arbitrary metric spaces, and so one can inquire about whether or not the invariant set of an IFS path has this condition regardless of the topological type of its invariant. If the invariant set of an IFS path fails to be an arc, then philosophically it should be easier to verify the bounded turning condition.

Problem 7.3. Give necessary and sufficient conditions for an IFS path $(\mathcal{S}, \gamma)$ that is not an IFS arc to have bounded turning in terms of the similarities in $\mathcal{S}$ alone.

Characterizing metric spaces that are quasisymmetrically equivalent to the standard two-sphere is an important problem in geometric group theory [5] and in the dynamics of rational mappings [6]. In [21, Meyer gave examples of metric spaces that are quasisymmetrically equivalent to the standard sphere but have non-integer Hausdorff dimension; these examples were called snowspheres and can be considered as a two-dimensional version of Rohde's snowflakes.

Problem 7.4. Can a large and concrete class of "IFS snowspheres", including many with unequal scaling ratios, be shown to be quasisymmetrically equivalent to the standard sphere? For such snowspheres, does the Hausdorff dimension determine the invariant set up to bi-Lipschitz equivalence?

The possible homeomorphism types for invariant sets of IFS paths include many specific fractals, such as the Sierpinski gasket, as shown in Section 4.2. It is also possible to realize the Sierpinski carpet as the invariant set of an IFS path (see Figure 3). The quasisymmetric geometry of the Sierpinski carpet also plays an important role in dynamics and geometric group theory [4. Perhaps viewing the carpet as an IFS path can yield some insight into this subject. 

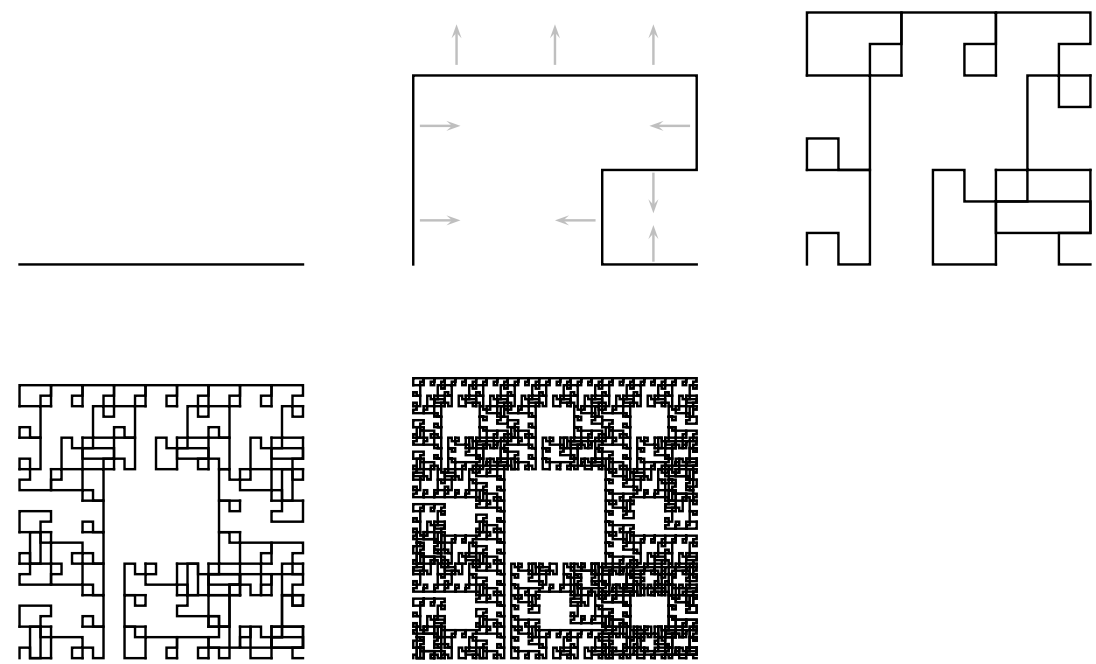

Figure 3. The sets $I$ and $T^{k}(I)$ for $k=1,2,3,4$ of an IFS path $\mathcal{S}=\left\{S_{1}, \ldots, S_{9}\right\}$ with the Sierpinski carpet as its invariant set. The gray arrows illustrate the orientation of the mappings $S_{i}$. Note that for this IFS path the similarity dimension equals $\frac{\log (9)}{\log (3)}=2$ where the Hausdorff dimension of the Sierpinski carpet is $\frac{\log (8)}{\log (3)}$.

\section{REFERENCES}

[1] V. V. Aseev, A. V. Tetenov, and A. S. Kravchenko, Self-similar Jordan curves on the plane (Russian, with Russian summary), Sibirsk. Mat. Zh. 44 (2003), no. 3, 481-492; English transl., Siberian Math. J. 44 (2003), no. 3, 379-386. MR.1984698

[2] Kari Astala, Personal communication.

[3] _ Self-similar zippers, Holomorphic functions and moduli, Vol. I (Berkeley, CA, 1986), Math. Sci. Res. Inst. Publ., vol. 10, Springer, New York, 1988, pp. 61-73. MR.955808 (89i:30018)

[4] M. Bonk, Quasiconformal geometry of fractals, International Congress of Mathematicians. Vol. II, Eur. Math. Soc., Zürich, 2006, pp. 1349-1373. MR2275649

[5] M. Bonk and B. Kleiner, Quasisymmetric parametrizations of two-dimensional metric spheres, Invent. Math. 150 (2002), no. 1, 127-183. MR.1930885

[6] Mario Bonk and Daniel Meyer, Expanding thurston maps, (preprint: arXiv:1009.3647).

[7] M. Bourdon and B. Kleiner, Combinatorial modulus, the combinatorial Loewner property, and Coxeter groups, Groups Geom. Dyn. 7 (2013), no. 1, 39-107. MR.3019076

[8] M. Carrasco Piaggio, On the conformal gauge of a compact metric space (English, with English and French summaries), Ann. Sci. Éc. Norm. Supér. (4) 46 (2013), no. 3, 495-548 (2013). MR3099984

[9] G. David and S. Semmes, Fractured fractals and broken dreams, Oxford Lecture Series in Mathematics and its Applications, vol. 7, The Clarendon Press, Oxford University Press, New York, 1997. MR.1616732

[10] K. J. Falconer, Dimensions and measures of quasi self-similar sets, Proc. Amer. Math. Soc. 106 (1989), no. 2, 543-554. MR969315

[11] K. J. Falconer and D. T. Marsh, Classification of quasi-circles by Hausdorff dimension, Nonlinearity 2 (1989), no. 3, 489-493. MR1005062

[12] K. J. Falconer and D. T. Marsh, On the Lipschitz equivalence of Cantor sets, Mathematika 39 (1992), no. 2, 223-233. MR.1203278 
[13] F. W. Gehring and K. Hag, The ubiquitous quasidisk, Mathematical Surveys and Monographs, vol. 184, American Mathematical Society, Providence, RI, 2012. With contributions by Ole Jacob Broch. MR2933660

[14] M. Ghamsari and D. A. Herron, Higher dimensional Ahlfors regular sets and chordarc curves in $\mathbf{R}^{n}$, Rocky Mountain J. Math. 28 (1998), no. 1, 191-222. MR.1639853

[15] M. Hata, On the structure of self-similar sets, Japan J. Appl. Math. 2 (1985), no. 2, 381-414. MR839336

[16] J. Heinonen, Lectures on analysis on metric spaces, Universitext, Springer-Verlag, New York, 2001. MR 1800917

[17] D. Herron and D. Meyer, Quasicircles and bounded turning circles modulo bi-Lipschitz maps, Rev. Mat. Iberoam. 28 (2012), no. 3, 603-630. MR2949615

[18] J. E. Hutchinson, Fractals and self-similarity, Indiana Univ. Math. J. 30 (1981), no. 5, 713747. MR625600

[19] M. Llorente and P. Mattila, Lipschitz equivalence of subsets of self-conformal sets, Nonlinearity 23 (2010), no. 4, 875-882. MR2602018

[20] J. McLaughlin, A note on Hausdorff measures of quasi-self-similar sets, Proc. Amer. Math. Soc. 100 (1987), no. 1, 183-186. MR883425

[21] D. Meyer, Snowballs are quasiballs, Trans. Amer. Math. Soc. 362 (2010), no. 3, 1247-1300. MR2563729

[22] Istvan Prause, Holomorphic motions: http://www.math.helsinki.fi/ prause/qc.html.

[23] H. Rao, H.-J. Ruan, and Y. Wang, Lipschitz equivalence of self-similar sets: algebraic and geometric properties, Fractal geometry and dynamical systems in pure and applied mathematics. I. Fractals in pure mathematics, Contemp. Math., vol. 600, Amer. Math. Soc., Providence, RI, 2013, pp. 349-364. MR3203409

[24] S. Rohde, Quasicircles modulo bilipschitz maps, Rev. Mat. Iberoamericana 17 (2001), no. 3, 643-659. MR 1900898

[25] P. Tukia and J. Väisälä, Quasisymmetric embeddings of metric spaces, Ann. Acad. Sci. Fenn. Ser. A I Math. 5 (1980), no. 1, 97-114. MR595180

[26] Z.-Y. Wen and L.-F. Xi, Relations among Whitney sets, self-similar arcs and quasi-arcs, Israel J. Math. 136 (2003), 251-267. MR1998112

[27] L.-F. Xi, Lipschitz equivalence of self-conformal sets, J. London Math. Soc. (2) 70 (2004), no. 2, 369-382. MR2078899

Mathematisches Institut, Universität Bern, Sidlerstrasse 5, CH-3012 Bern, SwitzerLAND

E-mail address: annina.iseli@math.unibe.ch

Department of Mathematical Sciences, Montana State University, P.O. Box 172400 Bozeman, Montana 59717

E-mail address: kevin.wildrick@montana.edu 